\title{
Change in Tetracene Polymorphism Facilitates Triplet Transfer in Singlet Fission-Sensitized Silicon Solar Cells
}

\author{
Benjamin Daiber, ${ }^{\nabla}$ Sourav Maiti, $\nabla$ Silvia M. Ferro, Joris Bodin, Alyssa F. J. van den Boom, \\ Stefan L. Luxembourg, Sachin Kinge, Sidharam P. Pujari, Han Zuilhof, Laurens D. A. Siebbeles,* \\ and Bruno Ehrler*
}

Cite This: J. Phys. Chem. Lett. 2020, 11, 8703-8709

Read Online

ACCESS | 네 Metrics \& More | 回 Article Recommendations | sl Supporting Information

ABSTRACT: Singlet fission in tetracene generates two triplet excitons per absorbed photon. If these triplet excitons can be effectively transferred into silicon ( $\mathrm{Si}$ ), then additional photocurrent can be generated from photons above the bandgap of Si. This could alleviate the thermalization loss and increase the efficiency of conventional Si solar cells. Here, we show that a change in the polymorphism of tetracene deposited on Si due to air exposure facilitates triplet transfer from tetracene into Si. Magnetic field-dependent photocurrent measurements confirm that triplet excitons contribute to the photocurrent. The decay of tetracene delayed photoluminescence was used to determine a transfer efficiency of $\sim 36 \%$ into Si. Our study suggests that control over the morphology of tetracene during the deposition will be of great importance to boost the triplet transfer yield further.

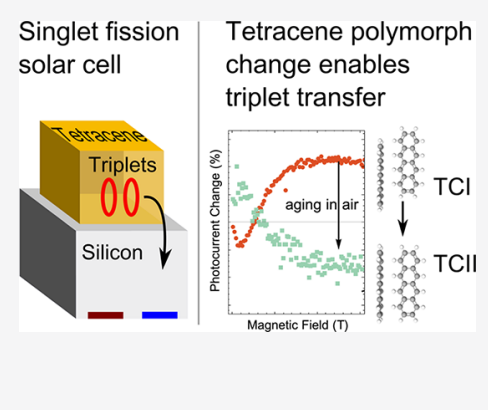

Silicon $(\mathrm{Si})$ is currently the dominating semiconductor $\checkmark$ material for solar cells but suffers from several loss mechanisms that reduce its efficiency. ${ }^{1,2}$ The largest loss mechanism results from the inefficient utilization of highenergy photons. The additional energy between the Si bandgap and the high-energy photons is lost to heat. Sensitizing Si solar cells with a top layer of singlet fission material can reduce this loss, and theoretically even overcome the Shockley-Queisser efficiency limit of $\sim 31 \%$ for a single-junction solar cell. ${ }^{3-14}$

Singlet fission is a spin-allowed process of creating two triplet excitons from one singlet exciton that can occur in certain organic semiconductor materials with delocalized $\pi$ orbitals. ${ }^{5,15-17}$ In this paper, we will focus on tetracene, which consists of four benzene rings that are annularly and linearly fused (Figure 1a). Upon absorption of a high energy photon $(>2.4 \mathrm{eV})$, a singlet exciton (bound electron-hole pair) is formed. This singlet exciton $\left(S_{1}\right)$ can subsequently be split into two triplet excitons $(\mathrm{T})$, each with roughly half the energy of the singlet exciton. This singlet fission process is mediated by a pair of spin-correlated triplets (TT), based on the kinetic model proposed by Johnson and Merrifield in 1970: ${ }^{18-20}$

$$
\mathrm{S}_{0}+h v \rightarrow \mathrm{S}_{1} \rightarrow(\mathrm{TT}) \rightarrow \mathrm{T}+\mathrm{T}
$$

where $S_{0}$ is the singlet ground state, $h \nu$ is the incoming photon energy, and $\mathrm{T}+\mathrm{T}$ denotes a pair of free triplets. In this model, the rate of singlet fission is determined by the coupling between the $S_{1}$ and TT states. ${ }^{21,22}$ Singlet fission competes with other processes (e.g., radiative and nonradiative recombination and excimer formation), such that some singlet excitons are lost and cannot undergo singlet fission. In tetracene, one absorbed photon leads to $\sim 2$ triplet excitons, because singlet fission is very fast, compared to other competing decay channels. ${ }^{23,24}$

In a solar cell architecture, where the energy of the triplet exciton is transferred to $\mathrm{Si}$, the bandgap of the $\mathrm{Si}$ cell must be smaller than the energy of the triplet exciton state of the singlet fission material. In tetracene, the triplet energy is $\sim 1.25 \mathrm{eV}$, which exceeds the $\mathrm{Si}$ bandgap of $1.1 \mathrm{eV}$, allowing triplet exciton transfer. ${ }^{24-27}$ The $V_{\text {oc }}$ value is determined by the lowbandgap semiconductor $\mathrm{Si}$, and the photocurrent from the high-energy photons can be doubled, because of singlet fission that eventually generates two electron-hole pairs from the high-energy photons (with an energy of $>2.4 \mathrm{eV}$ ).

Triplet transfer to Si can happen through energy transfer or charge transfer. In the case of energy transfer, both electrons and holes arrive in Si concurrently. However, if either electrons or holes are transferred to Si via charge transfer, the remaining countercharges in tetracene must be extracted by an additional contact. $^{26}$ Hence, if triplet energy transfer to Si could be realized, the resulting tetracene-Si solar cell would not need an additional charge-extracting electrode on top of the tetracene layer. Therefore, in principle, energy transfer could enable a simpler solar cell architecture and less added cost to $\mathrm{Si}$ solar cell manufacturing. Since the tetracene triplet energy is higher than the Si bandgap, transfer into $\mathrm{Si}$ is energetically

Received: July 15, 2020

Accepted: September 22, 2020

Published: September 22, 2020

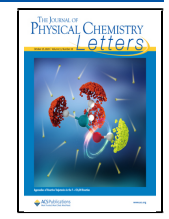




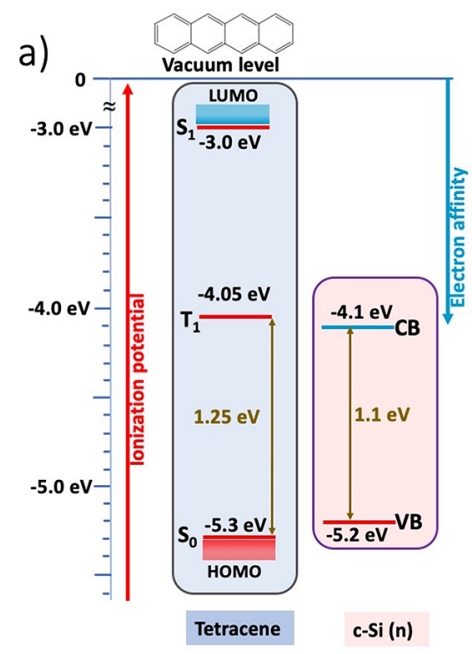

b)

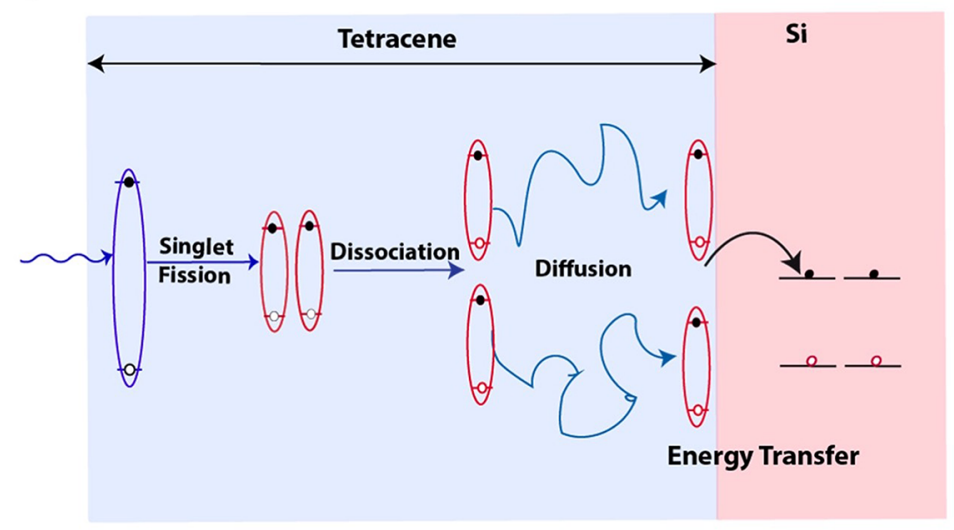

Figure 1. (a) Energy alignment of tetracene in the ground state $\left(S_{0}\right)$, triplet state $\left(T_{1}\right)$, singlet state $\left(S_{1}\right)$, and $S i$ valence band $(V B)$ and conduction band (CB) from the literature. ${ }^{24-26,28}$ The structure of tetracene is shown at the top. (b) Schematic of a singlet fission-sensitized Si solar cell. Photons at or above the singlet energy of tetracene are absorbed and create one singlet exciton, which splits into two triplet excitons forming a correlated triplet pair (TT) via singlet fission. The TT dissociates into free triplets, which can then independently diffuse to the tetracene/Si interface and transfer into $\mathrm{Si}$ to generate free-charge carriers.

a)

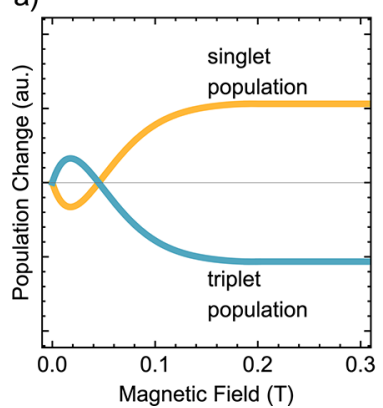

b)

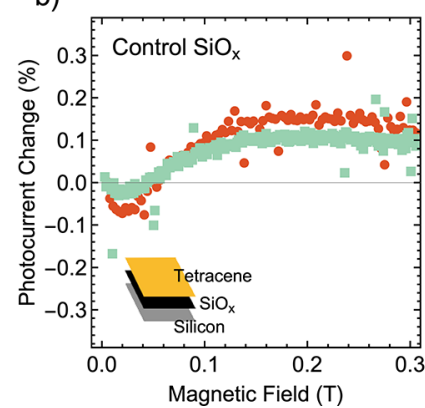

c)

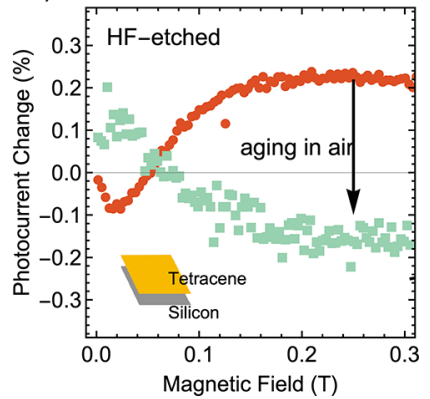

Figure 2. (a) Schematic for the behavior of singlet and triplet population in tetracene for photocurrent, as a function of the magnetic field. (b, c) Magnetic field-dependent photocurrent measurements for $\mathrm{Si}_{1} / \mathrm{SiO}_{x} /$ tetracene (control) (panel (b)) and HF-Si/tetracene (panel (c)), both before (red curve) and after (green curve) exposure to air. For both samples in panels (b) and (c), the positive change in photocurrent can be attributed to the dominant contribution of singlets. Aging the HF-etched sample in air flips the curve and leads to a triplet curve, indicating that triplets are transferred and contributing to the Si photocurrent.

allowed. Figure 1a shows the ionization energy of the tetracene exciton states and the position of the Si bands. The absolute energy level of the triplet exciton ionization energy, with respect to the vacuum level, is reported to be in the range between $-4.0 \mathrm{eV}$ and $-4.3 \mathrm{eV} \cdot{ }^{24,26}$ Figure $1 \mathrm{~b}$ shows a schematic of the processes involved in the operation of a singlet fission-sensitized Si solar cell, singlet generation, singlet fission, triplet diffusion, and triplet transfer.

To date, the transfer of triplet excitons from the singlet fission layer to the underlying low-bandgap semiconductor has proven to be the bottleneck for real-world applications. The extraction of triplets directly from tetracene into $\mathrm{Si}$ has been investigated by several research groups. Piland et al. did not find any evidence of triplet transfer from tetracene into Si upon direct deposition and with a $\mathrm{LiF}$ spacer. ${ }^{29}$ MacQueen et al. reported a small contribution of triplets to the photocurrent upon direct deposition of tetracene on a Si solar cell. ${ }^{26}$ The reasons for inefficient triplet transfer could be related to insufficient passivation of the Si surface and the weak coupling between the triplet exciton molecular orbitals and the electronic states in Si. Recently, Einzinger et al. unambiguously reported successful triplet transfer from tetracene into $\mathrm{Si}$ with
$75 \%$ efficiency after passivating the $\mathrm{Si}$ with a thin $(8 \AA)$ dielectric layer of hafnium oxynitride $\left(\mathrm{HfO}_{x} \mathrm{~N}_{y}\right)$ grown through atomic layer deposition (ALD). ${ }^{30}$ The ALD-grown interlayer passivates the $\mathrm{Si}$ surface and is thin enough to allow the transfer of triplets from tetracene into Si. However, this system is very sensitive to the exact interlayer thickness and composition, and the effect of the tetracene structure remains unclear. The transfer mechanism is still under debate and additional self-passivation effects complicate the interpretation.

Here, we report evidence for the triplet exciton transfer in a simpler system, from tetracene into bare $\mathrm{Si}$, after exposure of the tetracene layer to ambient air. We find signatures of triplet exciton transfer in magnetic field-dependent photocurrent measurements and a faster decay of the delayed photoluminescence $(\mathrm{PL})$ from tetracene, indicating triplet exciton quenching. We correlate these changes to a change in tetracene morphology, as seen in X-ray diffraction (XRD) spectra that show the conversion of polycrystalline tetracene from polymorph I (TCI) to polymorph II (TCII). ${ }^{31,32}$ We propose that the change of tetracene polymorph is important for the observed triplet transfer to Si solar cells. 

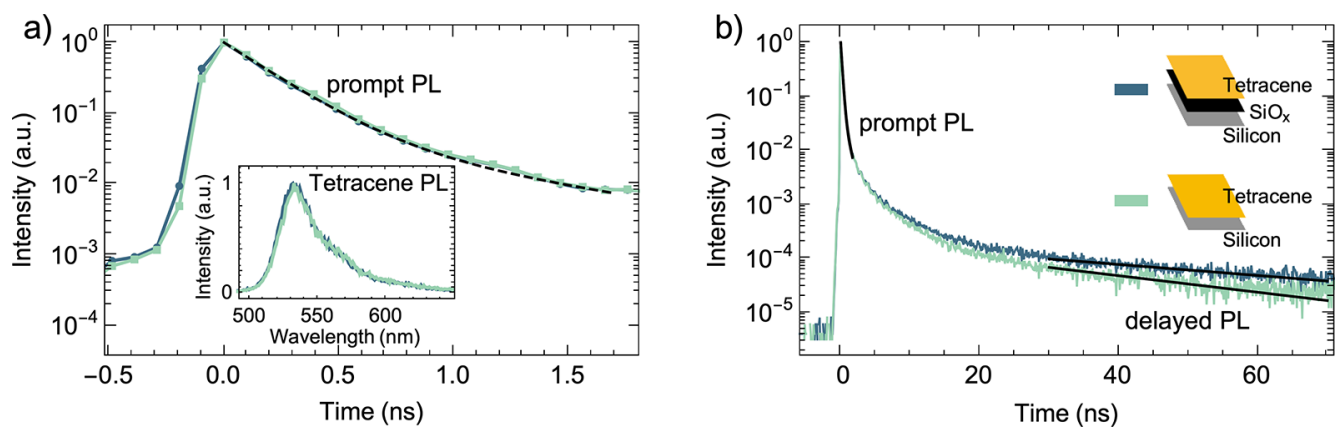

Figure 3. $\mathrm{PL}$ decay traces in $\mathrm{Si} / \mathrm{SiO}_{x} /$ tetracene and HF-Si/tetracene solar cells. Both samples have been aged in the air under similar conditions: (a) short-time (prompt) PL shows no difference in singlet fission time and efficiency between samples, and (b) long time (delayed) PL shows faster decay for the HF-Si/tetracene solar cell, which we attribute to triplet transfer to Si. The dotted black line represents the output from the kinetic model. The inset in panel (a) shows the PL spectra of both samples.

Measuring the effect of triplet excitons on the photocurrent of a solar cell is the most direct way of measuring the transfer of triplet excitons, and it is most relevant for the real-world application of a singlet fission-sensitized Si solar cell. The final goal is to increase the Si photocurrent from transferred triplet excitons. However, both singlet and triplet excitons can contribute to the photocurrent. Therefore, it is important to prove whether photocurrent originates from triplet versus singlet excitons. To distinguish between singlet and triplet exciton transfer, we exploit the behavior of singlet fission under a magnetic field (see Figure 2). Under a magnetic field of 300 $\mathrm{mT}$, singlet fission in tetracene becomes less efficient, resulting in a lower triplet exciton population, compared to the situation without the magnetic field. ${ }^{20}$ The characteristic shape of the photocurrent change under a magnetic field can be unambiguously attributed to triplet excitons originating from singlet fission. ${ }^{19,30,33}$ If the photocurrent from $\mathrm{Si}$ has the same magnetic field dependence as the triplet population (see the blue curve in Figure 2a), we conclude that there is transfer of triplet excitons to $\mathrm{Si}$. The photocurrent change is caused prevalently by triplet transfer; the opposite magnetic field dependence (yellow in Figure $2 \mathrm{a}$ ) would indicate that the photocurrent change is dominated by singlet transfer or radiative transfer. The relationship between magnetic field and singlet fission efficiency (or singlet/triplet populations) is not monotonic; below $50 \mathrm{mT}$, there is a small dip in the opposite direction, as described by Merrifield et al. (see also Figure 2a). ${ }^{20}$ This characteristic curve also allows us to exclude any other effects that the magnetic field could have on the photocurrent, such as displacement of the sample, induced currents at the contacts, or sample degradation over time.

We fabricated Si solar cells with an additional tetracene singlet fission top layer. The solar cells are heterostructure with intrinsic thin (HIT) layer solar cells with an interdigitated back contact (IBC). This means that contacts are on the back, which allows free access to the front surface. Where indicated, the solar cells are then encapsulated in an inert $\mathrm{N}_{2}$ atmosphere between two glass slides to keep oxygen and moisture out. Between the tetracene layer and the $\mathrm{Si}$ solar cell, we used different interlayers for reference measurements and to gain insight into the transfer mechanism. We then measured the photocurrent as a function of an externally applied magnetic field, as described above. Figure $2 \mathrm{~b}$ shows the magnetic field dependent photocurrent of solar cells with an insulating interlayer of $\sim 2 \mathrm{~nm} \mathrm{SiO}_{x}$, which shows no signature of triplet exciton transfer. A thick $(\sim 80 \mathrm{~nm}) \mathrm{Si}_{3} \mathrm{~N}_{4}(\mathrm{SiN})$ interlayer shows the same blocking behavior, as shown in Figure S1a in the Supporting Information. The photocurrent follows the curve that we would expect for singlet excitons, indicating that the singlet excitons contribute to the photocurrent. Utilizing a $\mathrm{HF}$ etch to remove the blocking layer and enabling direct contact between tetracene and $\mathrm{Si}$ (HF-Si/tetracene) does not change this behavior, as seen in Figure $2 c$ (red curve), which is consistent with earlier reports. ${ }^{29}$ The photocurrent still follows the singlet exciton population, and no evidence for triplet transfer is observed.

$\mathrm{Si} / \mathrm{SiO}_{x} /$ tetracene and $\mathrm{HF}-\mathrm{Si} /$ tetracene samples were then stored in the air under ambient conditions in the laboratory for 5 days and remeasured (see Figures $2 b$ and $2 c$ ). The magneticfield dependence of the photocurrent curve for the HF-Si/ tetracene solar cell, shown in Figure $2 c$, reverses for the airexposed sample, closely following the characteristic shape for a triplet exciton population, which is strong evidence for triplet exciton transfer. If we encapsulate the solar cell and store it in air, we also observe the triplet curve, although its emergence is then much slower, i.e., after 6 weeks, as shown in Figure S1b in the Supporting Information, indicating that, eventually, air enters the encapsulation. If the HF-Si/tetracene solar cell is stored under a dry nitrogen atmosphere in the glovebox $(<10$ ppm of $\mathrm{O}_{2},<1 \mathrm{ppm}$ of $\mathrm{H}_{2} \mathrm{O}$ ), we instead observed the singlet curve, which was retained after 6 weeks (Figure S1c in the Supporting Information). The strong difference in magneticfield photocurrent behavior between the air-exposed and nitrogen-stored samples indicates that air exposure plays a crucial role in enabling successful triplet transfer to Si. In Figure $2 c$, the decrease in photocurrent at high field is $\sim 0.2 \%$, which is comparable to $\mathrm{Si}-$ tetracene solar cells with $\mathrm{HfO}_{x} \mathrm{~N}_{y}$ interlayers. ${ }^{30}$ In that study, the self-passivation in $\mathrm{Si}$, because of improved surface screening by charge carriers at the $\mathrm{Si}$ interface, caused an increased photocurrent. ${ }^{30}$ This selfpassivation can lead to an overestimation of the contribution of triplet exciton injection, and the effects of triplet excitons and self-passivation were separated by a strong background illumination. We performed similar experiments to investigate the self-passivation of $\mathrm{Si}$ in our samples by using a strong (100 W) xenon light source with red light below the absorption onset of tetracene but above the absorption onset of Si. This allows us to inject charge carriers directly into $\mathrm{Si}$ that cannot have originated from tetracene. We did not see an influence of this additional light on the photocurrent change under the magnetic field, after correcting for the additional bias current, so we can exclude large influences from self-passivation in the 
Si solar cell (see Figure S2 in the Supporting Information). Therefore, we can conclude that exposure to air leads to triplet transfer from tetracene into Si. We also measured the external quantum efficiency (EQE) of the HF-etched solar cell and the same solar cell with tetracene before and after aging (see Figure S3 in the Supporting Information). After accounting for measurement position variation, we saw a small increase in EQE over the region of tetracene absorption, an increase likely within the noise level of our measurement. The tetracene layer is thicker than the triplet exciton diffusion length, and charge carriers are injected near the interface where the passivation is poor, leading to little additional photocurrent from triplet excitons. ${ }^{34}$ The following experiments will offer additional evidence for triplet exciton transfer and insight into the dynamics and mechanism.

To investigate the mechanism, time scale, and yield of the transfer process of triplets into $\mathrm{Si}$, we measured the tetracene PL decay both in the solar cells and tetracene deposited on $\mathrm{Si}$ wafers. The inset in Figure 3a, compares the PL spectra of $\mathrm{Si}$ / $\mathrm{SiO}_{x} /$ tetracene and $\mathrm{HF}-\mathrm{Si} /$ tetracene solar cells after exposure to air, showing the characteristic tetracene $0-0$ emission peak at $535 \mathrm{~nm}$ with a shoulder at $580 \mathrm{~nm}$ due to the $0-1$ transition, and a broad defect emission at $\sim 615 \mathrm{~nm}$. This defect emission arises from structural defects in tetracene during the vacuum evaporation process. ${ }^{35,36}$ The PL spectra are the same for both the samples upon air exposure, thus the PL spectra show no evidence of additional trap states from the aging process in samples with and without the $\mathrm{SiO}_{x}$.

The PL in tetracene originates from singlet exciton emission. The PL decay shows a fast initial component, because of singlet fission at short times ( $<1 \mathrm{~ns}$, prompt PL) and a longlived delayed PL arising from the triplet-triplet annihilation to singlet excitons at later times ( $>40 \mathrm{~ns}$, delayed $\mathrm{PL}$ ). The prompt PL decay is identical for the aged samples with and without the $\mathrm{SiO}_{x}$ blocking layer, showing that the singlet fission rate is not affected by the blocking layer (see Figure 3a). However, the delayed PL component was faster in the HF-Si/ tetracene solar cell upon air exposure, which provides additional evidence for the depopulation of triplets in tetracene caused by triplet transfer into $\mathrm{Si}$ (Figure $3 \mathrm{~b}$ ). Since triplet excitons disappear from tetracene, because they are transferred to $\mathrm{Si}$, triplet-triplet annihilation is reduced which, in turn, reduces the delayed PL intensity. The PL lifetime measurements have been reproduced with solar cell samples having a SiN blocking layer (see Figure S4 in the Supporting Information) showing no evidence for triplet transfer, just like in the $\mathrm{SiO}_{x}$ samples. The samples with and without the blocking layer in Figure $3 \mathrm{~b}$ and Figure S4 have been aged under similar conditions; the tetracene layer was exposed to air in all cases, but we only see delayed PL quenching in the sample without the interlayer. Therefore, we can exclude that oxygen quenching of the triplets leads to the faster delayed PL. The PL decay of samples with the SiN blocking layer and HFSi has been measured at different spots of the solar cell (see Figure S5 in the Supporting Information), and it showed little dependence on the measurement position on the samples. To reconfirm the results, PL decay measurements were performed in tetracene deposited on $\mathrm{Si}$ wafers (i.e., not a full solar cell) with and without the $\mathrm{SiO}_{x}$ blocking layer, and similar results were obtained (see Figure S6 in the Supporting Information). This ensures that the observed PL dynamics are a characteristic of the HF-Si/tetracene interface, and that it is not influenced by the presence of other solar cell components. Together with the magnetic field-dependent photocurrent measurements, we therefore correlate the faster decay in the delayed PL to triplet quenching, because of the triplet transfer process from tetracene to $\mathrm{Si}$.

What is the mechanism of activating triplet transfer in the HF-Si/tetracene samples after aging in the air? The activation could originate from either a change in the tetracene, or the $\mathrm{HF} / \mathrm{Si}$ interface, or both. We deployed X-ray diffraction (XRD) to detect changes in the tetracene morphology and Xray photoemission spectroscopy (XPS) to investigate changes on the HF-Si surface. Two different polymorphs, created by heated (TCI) or cooled (TCII) substrates, can form during tetracene deposition.

The TCII polymorph has different packing with increased distance along the $c$-axis, compared to TCI, resulting in a lower diffraction angle in XRD along the $(00 c)$ diffraction. ${ }^{32}$ Before air exposure, the XRD spectra show the presence of both polymorphs with slightly more TCI $\left(2 \Theta=7.3^{\circ}\right)$, compared to TCII $\left(2 \Theta=6.9^{\circ}\right)$, as seen in Figure 4 . However, after air

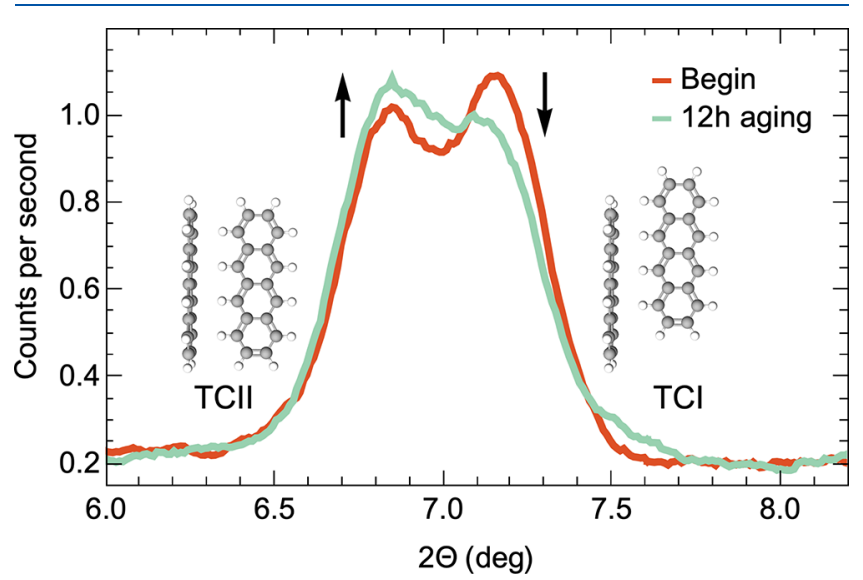

Figure 4. XRD of tetracene deposited on fresh HF-Si/tetracene and after $12 \mathrm{~h}$ of air exposure. We observe a conversion of TCI to TCII.

exposure, the ratio reversed with more TCII, compared to TCI, suggesting a change in polymorphism in tetracene. The two polymorphs have different intermolecular coupling strengths, because of a difference in molecular orientations that leads to a faster singlet fission rate in TCII, compared to TCI, as reported by Arias et al. ${ }^{31}$ The transition between both polymorphs is smooth (see Figure S7 in the Supporting Information) and is not triggered when storing the samples in nitrogen for seven months (see Figure S8 in the Supporting Information). We also observe a change in singlet fission rate, as the prompt PL decay of tetracene deposited on quartz becomes faster after air exposure (see Figure $S 9$ in the Supporting Information).

To confirm that the change responsible for triplet transfer is the aging of tetracene and not the aging of the HF-Si surface, we exposed HF-Si samples to air for different amounts of time, to grow a $\mathrm{SiO}_{x}$ layer with various thicknesses. We measured XPS to confirm the growth of this $\mathrm{SiO}_{x}$ overlay by monitoring the $\mathrm{Si}-\mathrm{O}_{x}$ peak in the $\mathrm{Si} 2 \mathrm{p}$ photoelectron emission narrow scan (see Figures S10a and S10b in the Supporting Information). On these samples, we deposited fresh tetracene and measured the PL decay. Triplet transfer was not observed in these samples (Figure S10c in the Supporting Information), confirming that triplet transfer is not associated with the 
growth of $\mathrm{a} \mathrm{SiO}_{x}$ layer, and the aging and subsequent change in the polymorphism of tetracene is related to the triplet transfer.

Triplet transfer via a direct Dexter-type mechanism is dependent on the overlap between the triplet exciton wave function of tetracene and of the electron and hole wave functions at the $\mathrm{Si}$ surface. ${ }^{37}$ This coupling will change, depending on the distance and orientation of the tetracene molecules, with respect to the Si surface. Therefore, the change in the orbital coupling in going from TCI to TCII and its effect on the triplet transfer efficiency is likely crucial and must be investigated further theoretically. A recent report by Niederhausen et al. that deployed near-edge X-ray absorption fine structure (NEXAFS), XPS, and density functional theory (DFT) calculations also suggests that different orientations at the interface exist and could lead to a change in transfer efficiency. ${ }^{38}$

To extract the triplet transfer rate and transfer efficiency, we model the PL decay data considering the singlet fission process, as depicted in Figure $5 \mathrm{a}^{29,31,39}$ The singlet fission
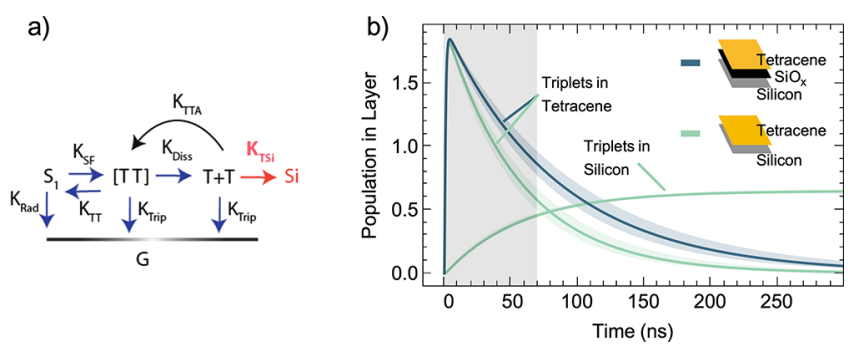

Figure 5. (a) Schematic representation of the kinetic model used to determine the triplet transfer efficiency; (b) the triplet population in $\mathrm{Si} / \mathrm{SiO}_{x} /$ tetracene (control), HF-Si/tetracene, and in HF-Si after air exposure predicted from our model, as a function of time. The gray area shows our experimental range of the PL decay measurements (70 ns). Transparent bands are $95 \%$ confidence intervals of the fitting parameters.

process in tetracene (with a rate $k_{\mathrm{SF}}$ ) competes with the radiative decay (with a rate of $k_{\mathrm{Rad}}$ ) through the formation of triplet pairs, which can then dissociate (at a rate of $k_{\text {Diss }}$ ) to form free triplets or fuse back (at a rate of $k_{\mathrm{TT}}$ ) to create an excited singlet state. The free triplets can decay with the triplet lifetime $\left(k_{\text {Trip }}\right)$ or regenerate the triplet pair state through triplet-triplet annihilation $\left(k_{\text {TTA }}\right)$. TTA results in the delayed PL from tetracene and determines the triplet lifetime. ${ }^{29,40,41}$ The populations of the $S_{1}$, TT, and T states can be determined by solving the coupled differential equations, as detailed in the Supporting Information. The $S_{1}$ population is plotted against the measured PL decay traces in Figure 3 (dotted lines) for the prompt and delayed PL. The rate constants described above were determined by solving the differential equation for HF$\mathrm{Si} /$ tetracene before and after exposure to air and using a leastsquares algorithm to fit the data. The rate constants obtained are consistent with the literature, as shown in Table S1 in the Supporting Information. The singlet fission time was determined to be $220 \pm 1 \mathrm{ps}$, which is in good agreement with reported values of $\sim 75-200 \mathrm{ps}$, depending on the crystallinity, grain size, and preparation conditions. $^{29,31,36,39,41,42}$ The faster decay of the delayed PL can be reproduced with the kinetic model by incorporating an additional triplet transfer process to $\mathrm{Si}$ without changing the other rate constants. The model based on these kinetic equations reproduces the data for prompt (until $1.7 \mathrm{~ns}$ ) and delayed PL (>30 ns) well, but fails to describe the decay at intermediate times between prompt and delayed PL. This is most likely due to additional effects of triplet pair diffusion, which have been explained by modeling that leads to a $t^{-3 / 2}$ dependence of the PL decay. ${ }^{43}$ Our intermediate PL decay data are also described by this function (see Figure S11 in the Supporting Information). ${ }^{43}$

From the kinetic model, the triplet lifetime is $85 \pm 6 \mathrm{~ns}$ in both samples and the triplet transfer time to $\mathrm{Si}$ is $169 \pm 8 \mathrm{~ns}$ in the aged sample. Figure $5 \mathrm{~b}$ shows how the triplet population varies with time with a faster decay of the triplet population in $\mathrm{HF}-\mathrm{Si}$, because of triplet transfer to $\mathrm{Si}$. The growth of the triplet population in HF-Si corresponds to $35.7 \% \pm 0.9 \%$ triplet transfer efficiency. Our model is robust against sampleto-sample variation, aging of tetracene on its own, and between wafers and solar cell (see the Supporting Information (Figures S4, S5, S6, and S9)). Our findings show that efficient triplet transfer can be obtained when the percentage of TCII is increased. Thus, to achieve even higher triplet transfer yield into HF-Si, control over the morphology appears to be crucial, so that TCII becomes the predominant polymorph. This trend suggests that, apart from the complex $\mathrm{HfO}_{x} \mathrm{~N}_{y}$ interlayers previously used, we can also exploit the tetracene orientation itself for efficient triplet transfer. ${ }^{30}$

In summary, we have shown that a change in the dominant tetracene polymorph by air exposure facilitates triplet transfer from tetracene into $\mathrm{Si}$. The triplet transfer process is confirmed through magnetic field-dependent photocurrent measurements, and the time scale of triplet transfer is obtained from delayed PL decay measurements. We find that the transition from the tetracene polymorph TCI to TCII is essential for efficient triplet transfer. This suggests that the orientation (with respect to the surface), and the packing of the singlet fission molecule is crucial for an efficient triplet transfer process. Future research should focus on an optimal alignment of tetracene molecules by preparing a pure TCII polymorph on $\mathrm{Si}$ and potential combinations of both interlayers and polymorph control, which could lead to the optimal triplet transfer efficiency. This could then enable the inexpensive manufacture of efficient singlet fission-sensitized Si solar cells.

\section{ASSOCIATED CONTENT}

\section{Supporting Information}

The Supporting Information is available free of charge at https://pubs.acs.org/doi/10.1021/acs.jpclett.0c02163.

Sample preparation and experimental details, additional magnetic field-dependent photocurrent measurements, EQE, XPS, additional XRD, and PL decay measurements (PDF)

\section{AUTHOR INFORMATION}

\section{Corresponding Authors}

Laurens D. A. Siebbeles - Optoelectronic Materials Section, Department of Chemical Engineering, Delft University of Technology, 2629 HZ Delft, The Netherlands; 이이.org/ 0000-0002-4812-7495; Email: 1.d.a.siebbeles@tudelft.nl

Bruno Ehrler - Center for Nanophotonics, AMOLF, 1098 XG Amsterdam, The Netherlands; O orcid.org/0000-0002-53073241; Email: b.ehrler@amolf.nl 


\section{Authors}

Benjamin Daiber - Center for Nanophotonics, AMOLF, 1098 XG Amsterdam, The Netherlands; (1) orcid.org/0000-00023773-1478

Sourav Maiti - Optoelectronic Materials Section, Department of Chemical Engineering, Delft University of Technology, $2629 \mathrm{HZ}$ Delft, The Netherlands; (1) orcid.org/0000-0003-1983-9159

Silvia M. Ferro - Center for Nanophotonics, AMOLF, 1098 XG Amsterdam, The Netherlands

Joris Bodin - Center for Nanophotonics, AMOLF, 1098 XG Amsterdam, The Netherlands

Alyssa F. J. van den Boom - Laboratory of Organic Chemistry, Wageningen University, 6708 WE Wageningen, The Netherlands

Stefan L. Luxembourg - TNO Energy Transition - Solar Energy, 1755 LE Petten, The Netherlands

Sachin Kinge - Toyota Motor Europe, Materials Research \& Development, B-1913 Zaventem, Belgium

Sidharam P. Pujari - Laboratory of Organic Chemistry, Wageningen University, 6708 WE Wageningen, The

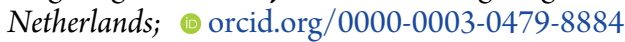

Han Zuilhof - Laboratory of Organic Chemistry, Wageningen University, 6708 WE Wageningen, The Netherlands; School of Pharmaceutical Science and Technology, Tianjin University,

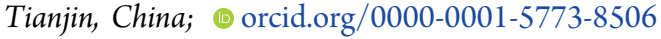

Complete contact information is available at: https://pubs.acs.org/10.1021/acs.jpclett.0c02163

\section{Author Contributions}

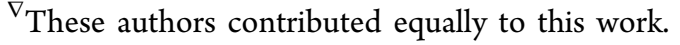

\section{Notes}

The authors declare no competing financial interest.

\section{ACKNOWLEDGMENTS}

This research received funding from The Netherlands Organization for Scientific Research (NWO) in the framework of the Materials for sustainability and from the Ministry of Economic Affairs in the framework of the PPP allowance.

\section{REFERENCES}

(1) Reese, M. O.; Glynn, S.; Kempe, M. D.; McGott, D. L.; Dabney, M. S.; Barnes, T. M.; Booth, S.; Feldman, D.; Haegel, N. M. Increasing Markets and Decreasing Package Weight for High-specificpower Photovoltaics. Nat. Energy 2018, 3 (11), 1002-1012.

(2) Ramanujam, J.; Verma, A.; González-Díaz, B.; Guerrero-Lemus, R.; del Cañizo, C.; García-Tabarés, E.; Rey-Stolle, I.; Granek, F.; Korte, L.; Tucci, M.; et al. Inorganic Photovoltaics - Planar and Nanostructured Devices. Prog. Mater. Sci. 2016, 82, 294-404.

(3) Shockley, W.; Queisser, H. J. Detailed Balance Limit of Efficiency of p-n Junction Solar Cells. J. Appl. Phys. 1961, 32 (3), $510-519$.

(4) Tayebjee, M. J. Y.; Gray-Weale, A. A.; Schmidt, T. W. Thermodynamic Limit of Exciton Fission Solar Cell Efficiency. J. Phys. Chem. Lett. 2012, 3 (19), 2749-2754.

(5) Smith, M. B.; Michl, J. Recent Advances in Singlet Fission. Annu. Rev. Phys. Chem. 2013, 64 (1), 361-386.

(6) Nelson, C. A.; Monahan, N. R.; Zhu, X. Y. Exceeding the Shockley-Queisser Limit in Solar Energy Conversion. Energy Environ. Sci. 2013, 6 (12), 3508-3519.

(7) Congreve, D. N.; Lee, J.; Thompson, N. J.; Hontz, E.; Yost, S. R.; Reusswig, P. D.; Bahlke, M. E.; Reineke, S.; Van Voorhis, T.; Baldo, M. A. External Quantum Efficiency Above $100 \%$ in a Singlet-ExcitonFission-Based Organic Photovoltaic Cell. Science 2013, 340 (6130), 334.
(8) Beard, M. C.; Johnson, J. C.; Luther, J. M.; Nozik, A. J. Multiple Exciton Generation in Quantum Dots versus Singlet Fission in Molecular Chromophores for Solar Photon Conversion. Philos. Trans. R. Soc., A 2015, 373 (2044), 20140412.

(9) Pazos-Outón, L. M.; Lee, J. M.; Futscher, M. H.; Kirch, A.; Tabachnyk, M.; Friend, R. H.; Ehrler, B. A Silicon-Singlet Fission Tandem Solar Cell Exceeding 100\% External Quantum Efficiency with High Spectral Stability. ACS Energy Lett. 2017, 2 (2), 476-480.

(10) Rao, A.; Friend, R. H. Harnessing Singlet Exciton Fission to Break the Shockley-Queisser limit. Nat. Rev. Mater. 2017, 2, 17063.

(11) Kunzmann, A.; Gruber, M.; Casillas, R.; Zirzlmeier, J.; Stanzel, M.; Peukert, W.; Tykwinski, R. R.; Guldi, D. M. Singlet Fission for Photovoltaics with 130\% Injection Efficiency. Angew. Chem., Int. Ed. 2018, 57 (33), 10742-10747.

(12) Futscher, M. H.; Rao, A.; Ehrler, B. The Potential of Singlet Fission Photon Multipliers as an Alternative to Silicon-Based Tandem Solar Cells. ACS Energy Lett. 2018, 3 (10), 2587-2592.

(13) Gish, M. K.; Pace, N. A.; Rumbles, G.; Johnson, J. C. Emerging Design Principles for Enhanced Solar Energy Utilization with Singlet Fission. J. Phys. Chem. C 2019, 123 (7), 3923-3934.

(14) Felter, K. M.; Grozema, F. C. Singlet Fission in Crystalline Organic Materials: Recent Insights and Future Directions. J. Phys. Chem. Lett. 2019, 10 (22), 7208-7214.

(15) Singh, S.; Jones, W. J.; Siebrand, W.; Stoicheff, B. P.; Schneider, W. G. Laser Generation of Excitons and Fluorescence in Anthracene Crystals. J. Chem. Phys. 1965, 42 (1), 330-342.

(16) Swenberg, C. E.; Stacy, W. T. Bimolecular Radiationless Transitions in Crystalline Tetracene. Chem. Phys. Lett. 1968, 2 (5), 327-328.

(17) Smith, M. B.; Michl, J. Singlet Fission. Chem. Rev. 2010, 110 (11), 6891-6936.

(18) Johnson, R. C.; Merrifield, R. E. Effects of Magnetic Fields on the Mutual Annihilation of Triplet Excitons in Anthracene Crystals. Phys. Rev. B 1970, 1 (2), 896-902.

(19) Groff, R. P.; Avakian, P.; Merrifield, R. E. Magnetic Field Dependence of Delayed Fluorescence from Tetracene Crystals. J. Lumin. 1970, 1-2, 218-223.

(20) Merrifield, R. E.; Avakian, P.; Groff, R. P. Fission of Singlet Excitons into Pairs of Triplet Excitons in Tetracene Crystals. Chem. Phys. Lett. 1969, 3 (3), 155-157.

(21) Miyata, K.; Conrad-Burton, F. S.; Geyer, F. L.; Zhu, X. Y. Triplet Pair States in Singlet Fission. Chem. Rev. 2019, 119, 42614292.

(22) Piland, G. B.; Burdett, J. J.; Dillon, R. J.; Bardeen, C. J. Singlet Fission: From Coherences to Kinetics. J. Phys. Chem. Lett. 2014, 5 (13), 2312-2319.

(23) Burdett, J. J.; Müller, A. M.; Gosztola, D.; Bardeen, C. J. Excited State Dynamics in Solid and Monomeric Tetracene: The Roles of Superradiance and Exciton Fission. J. Chem. Phys. 2010, 133 (14), 144506.

(24) Chan, W.-L.; Ligges, M.; Zhu, X. Y. The Energy Barrier in Singlet Fission Can Be Overcome through Coherent Coupling and Entropic Gain. Nat. Chem. 2012, 4, 840.

(25) Tomkiewicz, Y.; Groff, R. P.; Avakian, P. Spectroscopic Approach to Energetics of Exciton Fission and Fusion in Tetracene Crystals. J. Chem. Phys. 1971, 54 (10), 4504-4507.

(26) MacQueen, R. W.; Liebhaber, M.; Niederhausen, J.; Mews, M.; Gersmann, C.; Jäckle, S.; Jäger, K.; Tayebjee, M. J. Y.; Schmidt, T. W.; Rech, B.; et al. Crystalline Silicon Solar Cells with Tetracene Interlayers: The Path to Silicon-Singlet Fission Heterojunction Devices. Mater. Horiz. 2018, 5 (6), 1065-1075.

(27) Reineke, S.; Baldo, M. A. Room Temperature Triplet State Spectroscopy of Organic Semiconductors. Sci. Rep. 2015, 4 (1), 3797.

(28) Zhu, X. Y. How to Draw Energy Level Diagrams in Excitonic Solar Cells. J. Phys. Chem. Lett. 2014, 5 (13), 2283-2288.

(29) Piland, G. B.; Burdett, J. J.; Hung, T.-Y.; Chen, P.-H.; Lin, C.F.; Chiu, T.-L.; Lee, J.-H.; Bardeen, C. J. Dynamics of Molecular Excitons Near a Semiconductor Surface Studied by Fluorescence 
Quenching of Polycrystalline Tetracene on Silicon. Chem. Phys. Lett. 2014, 601, 33-38.

(30) Einzinger, M.; Wu, T.; Kompalla, J. F.; Smith, H. L.; Perkinson, C. F.; Nienhaus, L.; Wieghold, S.; Congreve, D. N.; Kahn, A.; Bawendi, M. G.; et al. Sensitization of Silicon by Singlet Exciton Fission in Tetracene. Nature 2019, 571 (7763), 90-94.

(31) Arias, D. H.; Ryerson, J. L.; Cook, J. D.; Damrauer, N. H.; Johnson, J. C. Polymorphism Influences Singlet Fission Rates in Tetracene Thin Films. Chem. Sci. 2016, 7 (2), 1185-1191.

(32) Schatschneider, B.; Monaco, S.; Tkatchenko, A.; Liang, J.-J. Understanding the Structure and Electronic Properties of Molecular Crystals Under Pressure: Application of Dispersion Corrected DFT to Oligoacenes. J. Phys. Chem. A 2013, 117 (34), 8323-8331.

(33) Wu, T. C.; Thompson, N. J.; Congreve, D. N.; Hontz, E.; Yost, S. R.; Van Voorhis, T.; Baldo, M. A. Singlet fission Efficiency in Tetracene-Based Organic Solar Cells. Appl. Phys. Lett. 2014, 104 (19), 193901.

(34) Akselrod, G. M.; Deotare, P. B.; Thompson, N. J.; Lee, J.; Tisdale, W. A.; Baldo, M. A.; Menon, V. M.; Bulović, V. Visualization of Exciton Transport in Ordered and Disordered Molecular Solids. Nat. Commun. 2014, 5, 3646.

(35) Voigt, M.; Langner, A.; Schouwink, P.; Lupton, J. M.; Mahrt, R. F.; Sokolowski, M. Picosecond Time Resolved Photoluminescence Spectroscopy of a Tetracene Film on Highly Oriented Pyrolytic Graphite: Dynamical Relaxation, Trap Emission, and Superradiance. J. Chem. Phys. 2007, 127 (11), 114705.

(36) Piland, G. B.; Bardeen, C. J. How Morphology Affects Singlet Fission in Crystalline Tetracene. J. Phys. Chem. Lett. 2015, 6 (10), 1841-1846.

(37) Dexter, D. L. A. Theory of Sensitized Luminescence in Solids. J. Chem. Phys. 1953, 21 (5), 836-850.

(38) Niederhausen, J.; MacQueen, R. W.; Lips, K.; Aldahhak, H.; Schmidt, W. G.; Gerstmann, U. Tetracene Ultrathin Film Growth on Hydrogen-Passivated Silicon. Langmuir 2020, 36 (31), 9099-9113.

(39) Wilson, M. W. B.; Rao, A.; Johnson, K.; Gélinas, S.; di Pietro, R.; Clark, J.; Friend, R. H. Temperature-Independent Singlet Exciton Fission in Tetracene. J. Am. Chem. Soc. 2013, 135 (44), 1668016688.

(40) Groff, R. P.; Avakian, P.; Merrifield, R. E. Coexistence of Exciton Fission and Fusion in Tetracene Crystals. Phys. Rev. B 1970, 1 (2), 815-817.

(41) Burdett, J. J.; Bardeen, C. J. The Dynamics of Singlet Fission in Crystalline Tetracene and Covalent Analogs. Acc. Chem. Res. 2013, 46 (6), 1312-1320.

(42) Zhu, T.; Wan, Y.; Guo, Z.; Johnson, J.; Huang, L. Two Birds with One Stone: Tailoring Singlet Fission for Both Triplet Yield and Exciton Diffusion Length. Adv. Mater. 2016, 28 (34), 7539-7547.

(43) Seki, K.; Sonoda, Y.; Katoh, R. Diffusion-Mediated Delayed Fluorescence by Singlet Fission and Geminate Fusion of Correlated Triplets. J. Phys. Chem. C 2018, 122 (22), 11659-11670. 